\title{
DEL PUNTAL AL CARACOL NUEVAS PERSPECTIVAS EN EL TIMBRE Y LA TÉCNICA DEL VIOLONCHELO*
}

FROM THE SPIKE TO THE SCROLL. NEW PERSPECTIVES IN THE TIMBRE AND TECHNIQUE OF THE CELLO

Sergio Andrés Castrillón Arcila**

Correo electrónico: sercello@gmail.com

Artículo recibido: 26-05-2019

Artículo aprobado: 12-07-2019

* Este artículo deviene de una investigación doctoral que estudia detalladamente el timbre y las técnicas extendidas en el repertorio para violonchelo de los siglos XX y XXI, titulada "New Timbral Directions in the Contemporary Cello Repertoire: Analysis of works by Colombian composers from 2000 to 2015", desarrollada en la Facultad de Artes de la Universidad de Helsinki.

** Compositor, violonchelista y Doctor en Musicología de la Universidad de Helsinki. 


\section{Resumen}

El presente artículo busca estudiar el gran cambio en el timbre y en las técnicas de interpretación que se ha dado en la música para violonchelo solo desde el siglo xx hasta nuestros días. Timbre, como una compleja cualidad sonora, empezó a explorarse más y a adquirir mayor importancia en el repertorio para violonchelo solo a principios del siglo xx. Sin embargo, esta exploración no se desarrolla sino hasta después de los años cincuenta, cuando el timbre se consolida como elemento primario dentro del repertorio. En primer lugar, se analizaron las nuevas exploraciones tímbricas en el violonchelo y su influencia en el desarrollo del repertorio a solo y con medios electrónicos en el siglo xx y xxi. Después de este análisis, se examinó cómo el cambio de paradigma tímbrico en las obras para violonchelo solo del siglo xx y principios del xxi está ligado principalmente a dos grandes acontecimientos que han evolucionado de manera simultánea durante los mismos siglos: el desarrollo de las llamadas técnicas extendidas y el surgimiento de diversas tendencias musicales.

Palabras clave: Timbre; Exploraciones tímbricas; Técnicas extendidas; Violonchelo solo; Música del siglo $\mathrm{xx}$.

\section{Abstract}

This article studies the change in the timbre and the playing techniques within the solo cello repertoire from the 20th century onward. Timbre as a complex sound element started to be more explored and to become more important in the solo cello literature in the beginning of the 20th century. However, this exploration was fully developed around 1950's when timbre is considered a crucial feature within the repertoire. First of all let us analyze the New Timbral Explorations in the cello and their influence in the solo cello and cello with electronic media repertoire in the 20th and 21st century. After this analysis, let us look how the paradigm shift in the timbre within the 20th and 21st centuries' solo cello repertoire is related to two main facts that have evolved simultaneously during such periods: The development of the so called Extended Playing Techniques and the emergence of diverse musical trends

Keywords: Timbre; Timbral explorations; Extended techniques; Cello solo; 20th century music. 


\section{Nuevas exploraciones tímbricas y repertorio en el siglo XX y XXI}

Después de establecerse como un importante instrumento solista ${ }^{1}$ desde su aparición, el violonchelo pareció no ser muy usado por los compositores dentro del repertorio a solo $^{2}$ durante el siglo xix y principios del siglo xx (Markevitch, 1989). Sumado a esto, y pese a los grandes cambios de paradigma en las técnicas de composición, así como en la construcción y el desarrollo físico que se dio en el instrumento durante la transición del siglo xix al xx, algunas de las piezas más importantes para violonchelo solo compuestas durante la primera mitad del siglo $\mathrm{xx}$ no presentan cambios tan rotundos con respecto al timbre y el tratamiento del sonido, como los que se dieron en muchas de las obras de la segunda mitad del siglo xx. En otras palabras, pese a que al mismo tiempo muchos compositores estaban entrando a explorar algunas de las tendencias musicales y artísticas de vanguardia, tales como el ruidismo, el serialismo y la música electrónica, mismas que empezaron a considerar el timbre como un elemento sobresaliente, había todavía un lugar para continuar componiendo bajo principios estéticos y estilísticos de tradición más larga. Dos claros ejemplos son las suites para violonchelo solo 1 en SoL mayor, 2 en RE menor y 3 en LA menor op. 131c (1915) de Max Reger, así como la sonata para violonchelo solo en Sol menor (1925) de Grandville Bantock.

Aun cuando estas piezas requieren de un alto nivel técnico y de expresión gestual por parte de los intérpretes, la mayoría de las técnicas sugeridas por ambos compositores eran ya parte de una larga tradición. Sin embargo, durante la misma época y hacia finales de la primera mitad del siglo $\mathrm{xx}$, varios compositores presentaron una perspectiva técnica y tímbrica diferente. Zoltán Kodály, en su sonata para violonchelo solo (1915), Gaspar Cassadó con la suite para violonchelo solo (1926) y Gyorgy Ligeti en su sonata para violonchelo solo (1948-1953) incluyeron escalas y ritmos usados en las músicas populares de sus países. Asimismo, trataron de recrear las sonoridades de instrumentos nacionales dentro del violonchelo. Por ejemplo, a lo largo de sus tres movimientos, la sonata de Kodály muestra una referencia al sonido y la interpretación del címbalo a través del uso de trinos en dobles cuerdas, trémolos, y pizzicatos percutidos, entre otras técnicas; Cassadó incluye en su suite una Sardana, donde, por medio de flautandos y armónicos naturales, el sonido del violonchelo toma un carácter más ligero, asemejándose al de un instrumento de viento (ver Ejemplo musical 2). Esto constituyó los primeros pasos de lo que Castrillón Arcila (2019) llamaría reinstrumentación, la cual hace parte de un proceso de re-significación tímbrica y del fenómeno de-construcción tímbrica.

Por otro lado, la sonata para violonchelo solo (1922) de Paul Hindemith explora cambios extremos de registro dentro de una organización de alturas que oscila entre un lenguaje tonal y atonal, dándole un carácter más abstracto al sonido del instrumento, algo no común dentro del repertorio para violonchelo solo de su época. Luego, a finales

Esto se refiere específicamente a piezas para violonchelo solo.

2 Se define como repertorio, en este caso, solo las obras, no los estudios técnicos. 
de la primera mitad del siglo xx, Luigi Dallapicolla en su Ciaccona, Intermezzo e Adagio (1947) para violonchelo solo, muestra ya claramente una constante interacción entre diferentes planos tímbricos a través de combinaciones de diferentes técnicas de arco y pizzicato, así como por medio del uso de cambios contrastantes de articulación y registro. A pesar de que en, estas piezas, elementos como forma, ritmo y altura seguían siendo los más importantes dentro del discurso musical, las contribuciones sonoras hechas por dichos compositores no fueron menores; de hecho, estas nuevas exploraciones tímbricas en el repertorio son claros antecedentes de los grandes cambios que se dieron en el uso del instrumento más adelante (ver Ejemplos musicales 1,2 y 3 ). ${ }^{3}$

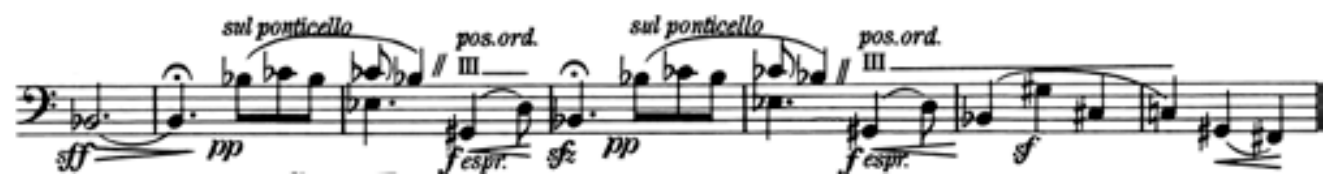

Ejemplo musical 1

Fragmento del primer movimiento de la sonata para violonchelo solo, op. 8. (1915) de Zoltán Kodály.

Vienna: Universal Edition, 1921

Obsérvese la interacción sucesiva entre sul ponticello y la posición ordinaria (T.E I), uno de los primeros ejemplos de modulación tímbrica en el repertorio para violonchelo solo del siglo $\mathrm{xx}$.

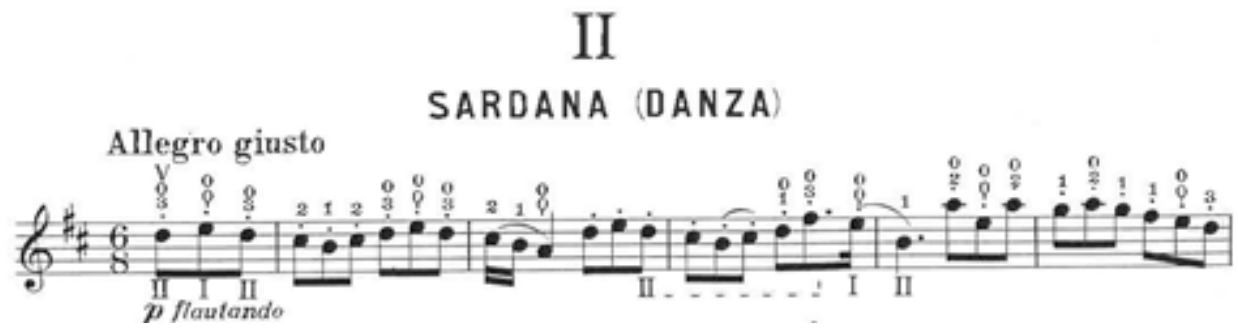

Ejemplo musical 2.

Pasaje del segundo movimiento, Sonata para violonchelo solo (1926)

Gaspar Cassadó.

Moscow: Muzyka, 1967. Plate 3972.

La obra yuxtapone dos técnicas extendidas: flautando (T.E I) y armónicos naturales (T.E III), en un registro agudo, con lo cual logra una sonoridad ligera y muy brillante. 


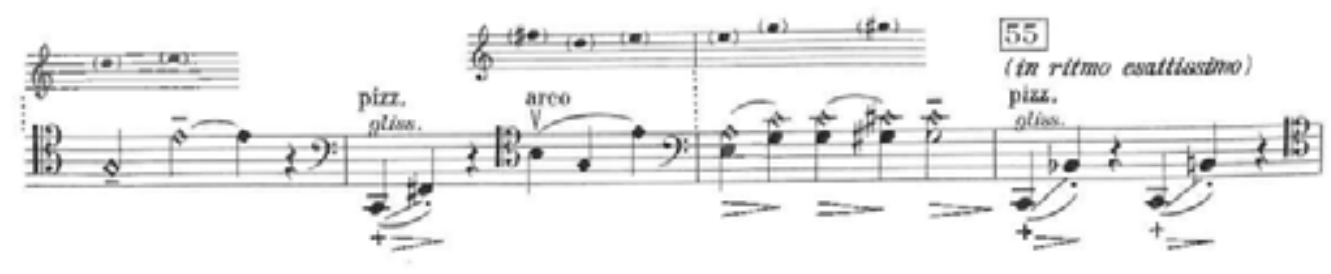

\section{Ejemplo musical 3. \\ Fragmento del primer movimiento de Ciaccona, Intermezzo e Adagio (1947) \\ Luigi Dallapicolla \\ Vienna: Universal Edition \\ Los armónicos artificiales (T.E III) y pizzicatos en glissando (T.E I), generan una especie de diálogo entre dos diferentes planos tímbricos.}

Durante la segunda mitad del siglo xx y como resultado de la aparición de diversos movimientos musicales, tales como la música concreta, la música electrónica, el indeterminismo y el experimentalismo, entre otros, muchas obras para violonchelo solo emergen repentinamente (Uitti, 2000). Estas obras no solo se caracterizaron por su novedad en forma y estructura, sino que también empezaron a re-significar el timbre del violonchelo. Si se considera Sonata para violonchelo solo (1957-1960) de Bernd Alois Zimmermann, Pression para un violonchelista (1969) de Helmut Lachenman, Kottos para violonchelo solo (1974) de Ianis Xenakis, Trilogía para violonchelo solo (1957-1964) de Giacinto Scelsi, Time and Motion Study II (1973-1976) para un violonchelista, su voz y electrónica de Bryan Ferneyhough, y Petals para violonchelo y electrónica (1988) de Kaija Saariaho, entre otras, dichas piezas incluyen el desarrollo de las llamadas técnicas extendidas, y aportan pruebas fehacientes de cómo la llegada de dispositivos electrónicos y la utilización del ruido, como elemento musical, influenciaron fuertemente a los compositores. Todos estos acontecimientos generaron nuevos procesos de composición que contribuyeron a un cambio de paradigma en el timbre y la técnica del violonchelo (ver Ejemplos musicales 4, 5, 6, 7 y 8).

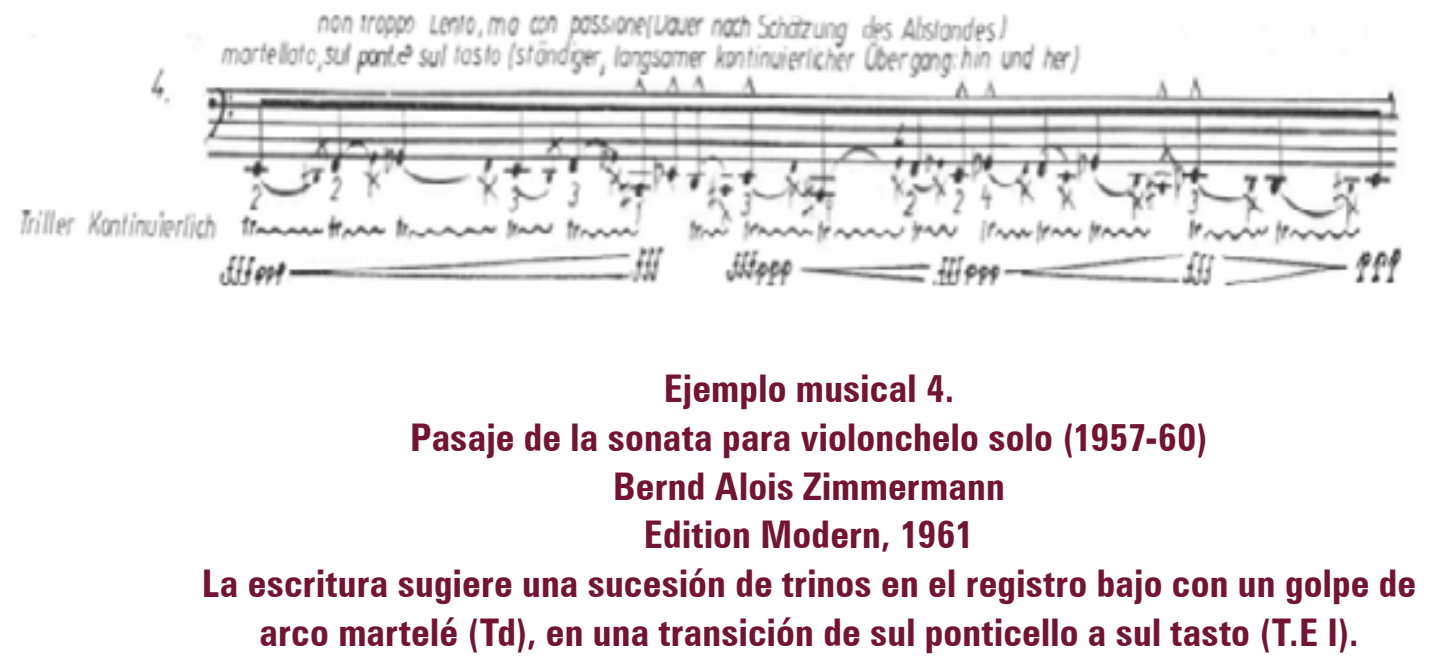


I.VIB. [MMU] (OSCILLAZIONE LARGA)

II.VIB. mMnmi (OSCILLAZIONE MEDIA)

III.VIB. (OSCILLAZIONESTRETTA)

\section{Ejemplo musical 5}

Trilogía para violonchelo solo (1957-64)

Giacinto Scelsi

Roma: Edizioni dell'autore

El autor sugiere un uso sistemático del vibrato con tres diferentes oscilaciones (T.E III).
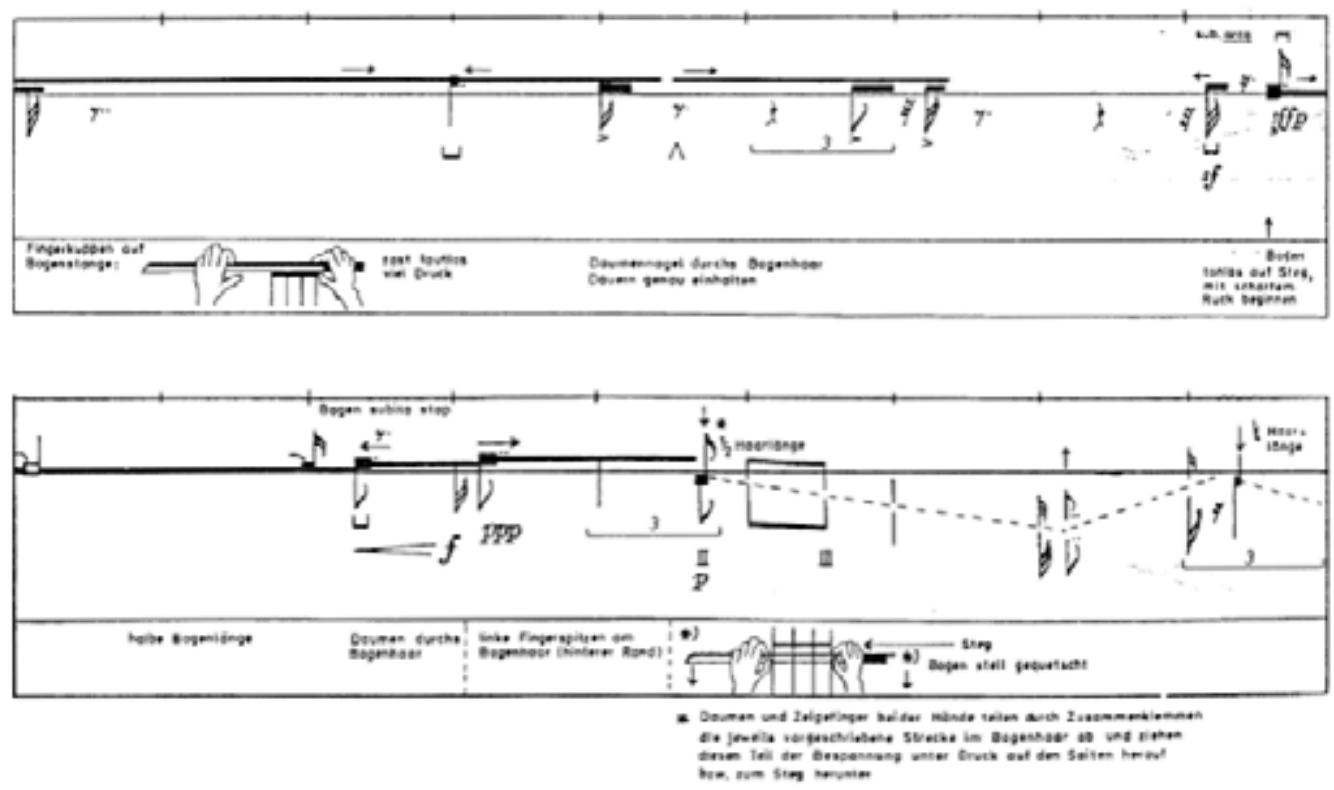

Ejemplo musical 6.

Pasaje de Pression, para un violonchelista (1969) de Helmut Lachenmann.

Breitkopf \& Härtel, 2012

El intérprete debe sostener el arco con la mano derecha y ponerlo sobre el puente, mientras que con la mano izquierda frota la madera del arco (T.E II). Esto genera una especie de amplificación de este gesto airoso, seguido por un desplazamiento del arco perpendicular a las cuerdas (T.E I). 
Durée - 8 min.

Duration -8 min.

Pour Violoncelle Seul

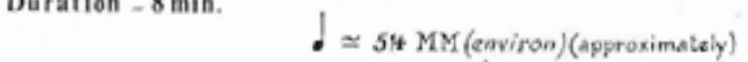

For Unaccompanied Violoncello

I. xénak is

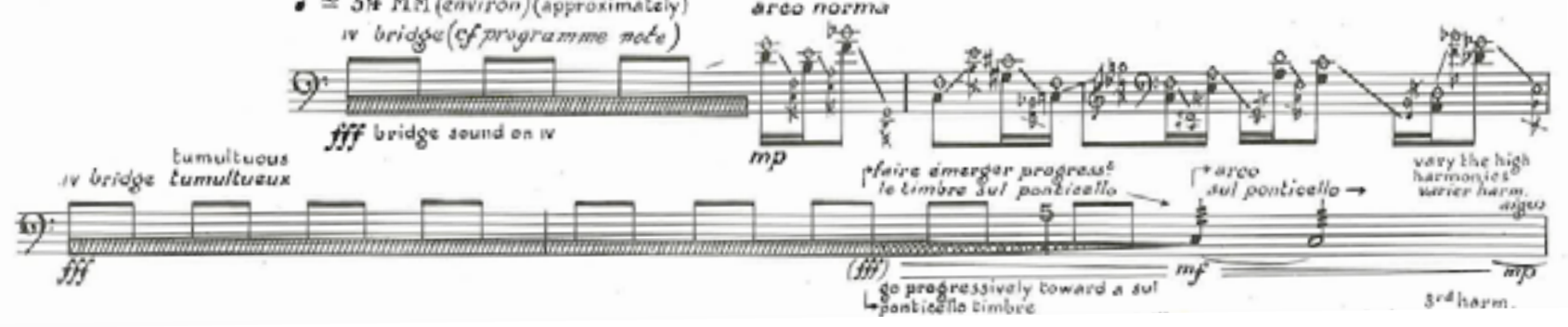

Ejemplo musical 7.

Kottos, para violonchelo solo (1974), de lannis Xenakis

Boosey \& Hawkes

Empieza con un fuerte sonido ruidoso sobre el puente en la cuarta cuerda (T.E II), intercalado con una rápida secuencia de armónicos artificiales (T.E III) que funcionan más como texturas de ruido que como alturas fijas.

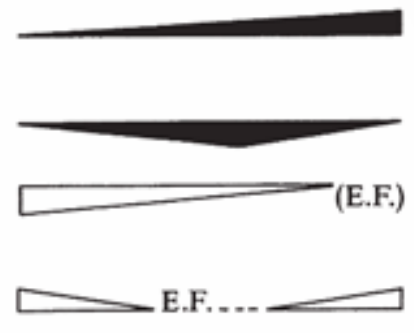

add bow pressure to produce a scratching sound, in which the audible pitch is totally replaced by the noise

as above but move back from noise to tone again

E.F.) decrease bow pressure to produce a soft, noise, windlike murmur

decrease bow pressure to produce a soft, noisy, winds-like murmur for as long as E.F. (estramamente flautando) continues, and move then gradually back to normal bow pressure

E.F. add bow pressure to produce a scratching sound, and decrease it gradually to produce the E.F. sound described above.

Ejemplo musical 8. Petals (1988), de Kaija Saariaho.

Edition Wilhelm Hansen, Helsinky

La obra pide un uso sistemático del ruido (T.E II) en el que deben ser usadas diferentes presiones de arco (T.E I) para lograr rangos específicos de tono y ruido.

Como consecuencia, los intérpretes empezaron a involucrarse mucho más en la creación y en la búsqueda de caminos sonoros alternativos, lo cual llevó al repertorio a diferentes direcciones y a expandir las maneras de interpretación. Al mismo tiempo, la estrecha relación entre intérpretes y compositores se consolidó más y más. Tal es el caso de Siegfried Palm, Rohan de Saram y Anssi Karttunen, quienes colaboraron con Bernd Alois Zimmermann, Mauricio Kagel, Iannis Xenakis, Kaija Saariaho y Magnus 
Lindberg, entre otros. Esto aparece junto al surgimiento de otras tendencias musicales tales como el jazz, el pop y el rock, entre otros, además del género free improvisation y la consolidación de la música experimental, donde el violonchelo fue y continúa teniendo gran importancia. Entre estos intérpretes se destacaron Charlotte Moorman, quien trabajó la música de John Cage, además de combinar su quehacer musical con las artes visuales y el performance; y Frances Marie Uitti, extensamente dedicada a la práctica de la improvisación, la composición, el desarrollo de nuevas técnicas de interpretación y la investigación tecnológica del violonchelo. Por otro lado, Abdul Wadud, Marcio Mattos, Tom Cora y Tristan Honsinger, como pioneros en la utilización del violonchelo en el free jazz y la free improvisation, también contribuyeron a dar al instrumento una nueva identidad (Castrillón Arcila, 2019). Sumado a esto, la combinación del violonchelo y el dispositivo electrónico, visto no como un dúo sino como una unidad, también fue un hecho importante que permitió el desarrollo técnico y tímbrico, además de la expansión y el crecimiento del repertorio, intensamente desarrollado desde la década de 1960 no solo en Europa, sino en todos los continentes (Stowell, 2000). Un trabajo importante que demuestra lo anteriormente dicho es la compilación de Homuth (1994), que muestra más de 1500 piezas para solo cello y cello con medios electrónicos pregrabados compuestas entre 1960 y 1993.

Otro punto importante es la contribución al aumento del repertorio para violonchelo solo y con medios electrónicos por parte de compositores latinoamericanos durante la segunda mitad del siglo xx. Esto constituyó un punto de referencia no solo histórico, sino un cambio en el paradigma en el timbre y la técnica del instrumento. La inclusión del dispositivo electrónico, el uso del ruido como material musical y la influencia de elementos de las músicas populares e indígenas generaron nuevas sonoridades y técnicas en el instrumento. Estas nuevas obras, emergentes de un continente prácticamente en silencio dentro del contexto de la vanguardia musical de la primera mitad del siglo xx, también contribuyeron a cuestionar y a expandir el rol del intérprete (Castrillón Arcila, 2016). Dentro de las principales obras se encuentran Synchronisms 3 (1965) y A7 (1966) para violonchelo y medios electrónicos pregrabados de Mario Davidovsky e Hilda Dianda, respectivamente, Siegfried, para violonchelo solo (1971) de Mauricio Kagel, Puneña, para violonchelo solo (1976) de Alberto Ginastera, Canto alterno (1978) y Yuunoihui'yei (1983) para violonchelo solo de Julio Estrada, y Llorando silencios, para violonchelo solo (1988) de Alejandro Iglesias Rossi, entre otras.

En la transición del siglo xx y xxI, compositores de diferentes latitudes empezaron a trabajar cada vez más en obras para violonchelo solo y violonchelo con medios electrónicos, muestra de un gran crecimiento en el repertorio mundial. Sin embargo, en Sudamérica se empezó a notar un desarrollo del repertorio mucho más rápido y más amplio, comparado con épocas anteriores. Dentro de muchas de las obras compuestas en este periodo se exponen varias características especiales.

Un cambio radical de paradigma en el timbre: en otras palabras, las nuevas exploraciones tímbricas que emergieron de las primeras obras compuestas en el siglo xx, que fueron desarrollándose hacia la segunda mitad del mismo, ahora han 
re-significado el timbre del instrumento y han generado otra identidad sonora en el mismo. Es decir, el fenómeno de re-instrumentación, el uso del ruido como material musical, las preparaciones y modificaciones del instrumento y otras manifestaciones, no son ya extraños a la sonoridad que el violonchelo solía ofrecer al repertorio. Por el contrario, la identidad sonora del instrumento parece haberse expandido a tal punto que, dentro del repertorio actual, el timbre y las técnicas para las cuales el violonchelo fue concebido no son ya las más utilizadas. Entre algunas de las obras del repertorio de este periodo están: Advaya para violonchelo, electrónica y sintetizador (1994) de Jonathan Harvey; Sequenza XIV, para violonchelo solo (2002) de Luciano Berio; Limit of correction, para violonchelo solo (2009) de Kristian Ireland; Desde las entrañas del Sudtrópico (2010), Detrás de la Montaña (2011-2012) y Yo soy la selva (2014), para violonchelo solo de Sergio Castrillón; Kärlek Splittring I, para violonchelo solo (20052008) y Kärlek Splittring II, para violonchelo con dos arcos y proceso electrónico en tiempo real (2013) de Marcos Suárez Cifuentes (ver Ejemplo musical 9).

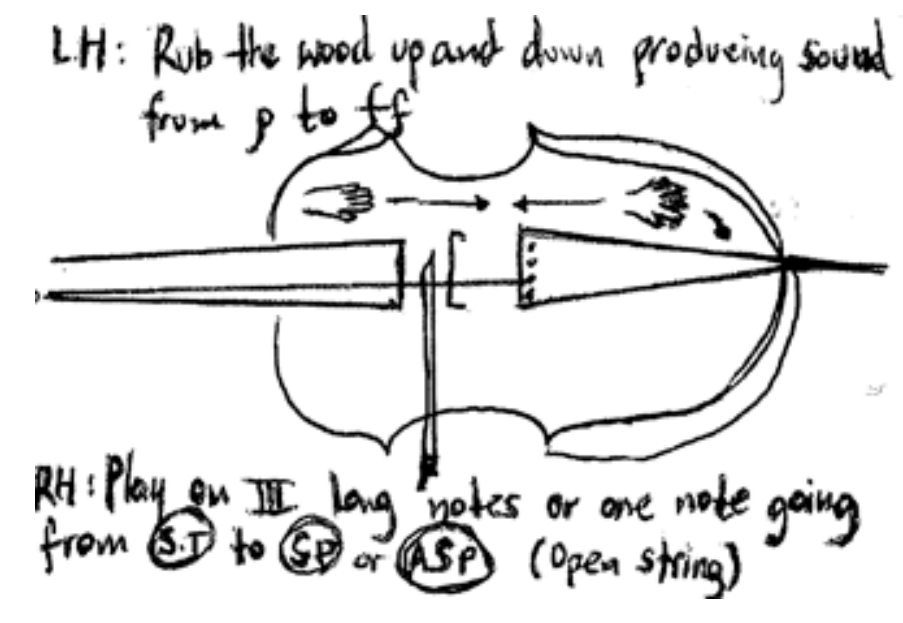

Ejemplo musical 9.

Fragmento de Yo soy la selva, para violonchelo solo (2014), de Sergio Castrillón. Partitura edita por el compositor

\section{La imagen muestra una yuxtaposición de dos gestos musicales: una modulación tímbrica lograda a través de cambios en la posición de arco sobre la tercera cuerda (T.E I); y un sonido frotado con la mano izquierda sobre la tapa frontal, con lo que se consigue una especie de polífonia de timbres.}

Obras multimedia, performáticas, conceptuales y con procesos en tiempo real: las colaboraciones interdisciplinarias -en el arte, la ciencia e incluso en ciertas prácticas físico-espirituales, tales como el yoga y el tai chi, entre otras-, además del uso del nuevas tecnologías han permitido que el repertorio para violonchelo solo y con medios electrónicos en el siglo xxI se encuentre en un constante desarrollo. De hecho, el formato "violonchelo a solo" es muchas veces confuso, puesto que, si bien en muchas 
ocasiones dichas obras recurren al violonchelo como única fuente acústica, otros medios actúan dentro de la misma pieza. Estas colaboraciones también influyen en los procesos de composición e interpretación y generan cambios en ellos. Por ejemplo, la notación musical tradicional no es más una herramienta para asegurar la total efectividad al trabajar obras que incluyen diferentes artes o procesos creativos en tiempo real, como la improvisación o el live electronics. De ahí que los compositores empezaron a generar otro tipo de partituras e incluso diferentes interfaces para la interpretación. Además, al trabajar con diferentes tipos de artistas, los intérpretes se vieron directamente involucrados en procesos de creación, muchas veces instantáneos, que empezaron a ser abordados desde una perspectiva más bien heurística. Esto marcó no sólo una diferencia en los procesos y resultados artísticos de dichas obras colectivas, sino que constituyó también un punto de quiebre en lo que solía ser el rol del compositor, del artista, del creador, del improvisador y del intérprete. Así se empieza a generar una especie de nueva figura que ya no tiene un rol específico, sino más bien multidisciplinario. Entre las piezas que muestran las características recién mencionadas es importante destacar Planos frotados, para violonchelo solo y acción performática (2009) de Esteban Agosín; Rellenos de la inaprehensible mostración: Otra metáfora, para violonchelo solo, medios electrónicos analógicos y recitación (2010) de Juan Manuel Cerono; Ashtánga, para violonchelo, video y procesos electrónicos en tiempo real (2010) de Matías Uribe; María ópera expandida para violonchelo eléctrico solista, video y electrónica procesados en tiempo real (2010-2011) de Héctor Fabio Torres, y ENNA IRA MA, para violonchelo, medios electrónicos y acción performática (2011) de Sergio Castrillón.

Desde finales de los años noventa, las obras para violonchelo solo y con medios electrónicos habían ya empezado a mostrar diversas estéticas y estilos musicales en ellas. Es decir, ya no sólo los compositores utilizaban el lenguaje musical de las tendencias musicales de vanguardia que habían empezado desde los años cincuenta; por el contrario, muchas de estas piezas tendían a rechazar dichas tradiciones y más bien incluían elementos del rock, jazz y de las llamadas músicas del mundo, entre otras. Entre algunos ejemplos de lo anterior destacan Trilogía para violonchelo solo (2002-2003) de Sergio Castrillón; E-nucleo vita aeternus, para violonchelo solo (2011) de Walter Barrera; Sed (2009), Violonchelo procesado (2011) y Vernecular (2013), para violonchelo eléctrico y pedales, de Nicolay Vassilev. Estas características han llevado a que el repertorio para violonchelo del siglo xxi sea amplio y ecléctico, además de que han contribuido al surgimiento de nuevos intérpretes interesados en expandir la identidad del instrumento a través de diferentes prácticas musicales y artísticas en general. Sin embargo, en esta época no es fácil para los compositores acceder a publicaciones por medio de editoriales o sellos discográficos reconocidos, lo cual de alguna manera hace que sus obras no adquieran la suficiente relevancia dentro del mundo académico musical. A su vez, los formatos impresos y de grabación no bastan por lo general para alcanzar una difusión o registro acertado de las obras. Es por eso que los creadores e intérpretes actuales se ven obligados a explorar constantemente diferentes plataformas para difundir sus obras y así poder comunicarse e interactuar 
con otros artistas, como se puede observar en la utilización del violonchelo en forma horizontal por Ernst Reijseger o de manera similar en algunas ocasiones por los integrantes de la banda de rock Apocalyptica.

\section{Técnicas extendidas en el violonchelo y diversas tendencias musicales}

Si bien es difícil calcular la cantidad de técnicas extendidas desarrolladas en el violonchelo durante los siglos xx y XxI, hay un rango que ha sido el más utilizado por compositores dentro del repertorio y que de alguna manera ha estandarizado ciertas sonoridades que hoy hacen parte de la tradición técnica del violonchelo contemporáneo. Aún en géneros como la free improvisation, el free jazz y el rock, donde el intérprete ha prácticamente generado por medio de una tradición no escrita nuevas técnicas de interpretación, el rango de sus aportaciones es mucho más amplio, a la vez que muy específico tímbricamente. Antes de analizar algunos ejemplos musicales que incluyen técnicas extendidas y sus características sonoras, es importante tener una aproximación al desarrollo de las técnicas fundamentales y su relación con la aparición de nuevas técnicas y tendencias musicales. Al mismo tiempo, es necesario introducir ciertas denominaciones que serán expuestas más adelante en este texto, dentro de una tabla de categorización.

Desde su aparición como instrumento solista hasta nuestros días, el violonchelo se ha usado principalmente a través de dos técnicas fundamentales, una especie de doble raíz: arco y pizzicato. Por lo general, las dos están destinadas a la mano derecha, aunque la mano izquierda ha cumplido la función de presionar las cuerdas, para definir las alturas cuando no se usan cuerdas al aire. Aun así, el desarrollo y las funciones técnicas de ambas manos han tomado nuevas direcciones a través del tiempo, debido a la aparición de nuevas piezas y nuevos intérpretes. Es importante analizar aquí por qué la técnica de arco fue más usada y desarrollada que el pizzicato en el repertorio solista compuesto antes del siglo xx. Esto se puede notar desde un punto de vista acústico, estilístico y de funcionalidad. Por ejemplo, el rango de resonancia que permiten las cuerdas frotadas en general cuando se usa el arco es mayor que con el pizzicato, además de que esta característica acústica se ligaba a cierta estética musical. Es decir, que el uso del arco permitía ejecutar más fácil y claramente las melodías, los acordes, así como los diferentes rangos dinámicos y de velocidad que requería la música para violonchelo solista. Es por eso que entre los siglos xviii y xix se desarrollaron las más importantes técnicas derivadas (usadas hasta nuestros días) ${ }^{4} \mathrm{y}$ diversos golpes de arco, presentes en diferentes obras y estudios técnicos para violonchelo. ${ }^{5}$ El uso del pizzicato,

4 Se usa esta denominación para referirme a ciertos golpes de arco y técnicas derivadas de la doble raíz (arco y pizzicato). Ver Tabla 1.

5 La contribución de violonchelistas compositores, como Luigi Boccherini, Jean-Louis Duport, Sebastian Lee y David Popper, entre muchos otros, fue fundamental para el desarrollo de la técnica de arco.

No. 11. Enero - junio 2019 
por su parte, era más limitado en cuanto a resonancia, especialmente en el registro agudo y sobre agudo, y no permitía ejecutar melodías rápidas con suficiente claridad. Pese a la facilidad con la que el pizzicato puede ser aplicado a acordes, arpegios y a diferentes rangos dinámicos, la concepción misma del instrumento y el estilo musical en estos periodos pareció no necesitar demasiado de las características sonoras que esta técnica podía ofrecer a las obras solistas. Más bien, el pizzicato en el violonchelo estaba reservado para algunos estudios técnicos, para la música de cámara y la orquestal, donde su función era más bien programática, es decir, que muchas veces buscaba evocar onomatopeyas y efectos sonoros que venían de ideas extramusicales. De ahí que podamos decir que, de alguna manera, el pizzicato se establece como la primera técnica extendida en el violonchelo. Mientras que la técnica de la mano derecha ampliaba sus posibilidades, la técnica de la mano izquierda experimentaba diversos cambios, no solo en la posición y uso de la presión, sino también en el nivel de complejidad armónica y el uso del registro agudo y sobreagudo que demandaban las piezas a solo. Por ejemplo, el uso de dobles cuerdas, arpegios y acordes empezó a ser casi fundamental dentro de las obras y los estudios solistas, lo cual promovió al violonchelo a la categoría de un instrumento armónico y, por ende, a generar más dificultades para la mano izquierda.

Durante el siglo $\mathrm{xx}$, y como consecuencia de la aparición de diversas tendencias musicales con diferentes principios estéticos, las técnicas derivadas mencionadas anteriormente empiezan a generar las técnicas extendidas sobre las cuerdas y las técnicas extendidas fuera de las cuerdas. Estas también influyeron en las funciones que tenía cada mano, especialmente la izquierda, que empezó a usarse para diferentes tipos de pizzicatos y otras técnicas derivadas de éste -el tapping por ejemplo-. Vale la pena mencionar que el desarrollo del pizzicato en los siglos xx y xxi ha llegado a ser tan sistemático y complejo como lo fue el del arco en los períodos previos. Sin embargo, la evolución de la técnica del arco y su uso en los siglos xx y xxi continúa siendo mayor que la del pizzicato y de otras técnicas que fueron surgiendo -percusión y frotación-. Todo esto indica que la multiplicación de tendencias musicales en el siglo xx generó la extensión de las técnicas del violonchelo, y estas nuevas técnicas fueron estableciendo a su vez características tímbricas que de algún modo empezaron a definir ciertas tendencias musicales. Si bien es complejo saber con exactitud cuándo aparecen y son utilizadas muchas de las técnicas que muestra la siguiente tabla, las referencias cronológicas que se muestran se conectan al tiempo en el que ellas fueron más exploradas y desarrolladas dentro del repertorio para violonchelo solo y por violonchelistas de diferentes géneros y tradiciones musicales. 


\begin{tabular}{|c|c|}
\hline \multicolumn{2}{|c|}{ Técnicas principales (doble raíz) } \\
\hline $\operatorname{arco}$ & pizzicato \\
\hline $\begin{array}{r}\text { Técnicas } \\
\text { (Principalmente usada }\end{array}$ & $\begin{array}{l}\text { derivadas } \\
\text { s en los siglos XVIII-XIX) }\end{array}$ \\
\hline $\begin{array}{l}\text { Articulación (golpes de arco) } \\
\text { - Detaché } \\
\text { - Martelé } \\
\text { - Legato } \\
\text { - Jeté } \\
\text { - Staccato } \\
\text { - Collé } \\
\text { - Louré } \\
\text { - Ricochet } \\
\text { - Sautillé } \\
\text { - Spiccato } \\
\text { - Tremolo }\end{array}$ & $\begin{array}{l}\text { Articulación (pizzicato con mano } \\
\text { derecha) } \\
\text { - Staccato } \\
\text { - Legato }\end{array}$ \\
\hline $\begin{array}{l}\text { Posición } \\
\text { — M.O (Modo ordinario) }\end{array}$ & $\begin{array}{l}\text { Posición } \\
-\quad \text { M.O (Modo ordinario) }\end{array}$ \\
\hline $\begin{array}{l}\text { Presión } \\
-\quad \text { Normal }\end{array}$ & $\begin{array}{l}\text { Presión } \\
-\quad \text { Normal }\end{array}$ \\
\hline $\begin{array}{l}\text { Oscilación de altura y expresión } \\
\text { (arco + mano izquierda) } \\
\text { - Vibrato moderado } \\
\text { - Trino de medio tono } \\
\text { - Glissando moderado (distancias } \\
\text { inteválicas pequeñas) } \\
\text { - Scordatura (en una o dos cuerdas) }\end{array}$ & $\begin{array}{l}\text { Oscilación de altura y expresión } \\
\text { (pizzicato + mano izquierda) } \\
\text { - Vibrato moderado } \\
\text { - Trino de medio tono } \\
\text { - } \text { Glissando moderado (distancias } \\
\quad \text { interválicas pequeñas) } \\
\text { - Scordatura (en una o dos cuerdas) }\end{array}$ \\
\hline
\end{tabular}


Técnicas principales (doble raíz)

Técnicas extendidas I

sobre las cuerdas

(T.E I)

(Principalmente usadas en los siglos XX-XXI)

Articulación (golpes de arco)
- Detaché
- Martelé
- Legato
- Jeté
- Staccato
- Collé
- Louré
- Ricochet
- Sautillé
- Spiccato
- Tremolo
- Arco circular
- Arco perpendicular
- Col legno
- Col legno battuto
Posición

- M.O/Pos. Ord (Modo ordinario/ Posición ordinaria)

- S.P (Sul ponticello)

- A.S.P (Alto sul ponticello)

- S.T (Sul tasto)

- A.S.T (Alto sul tasto)

- Sobre el puente

- Detrás del puente

- Detrás de la ceja

- A la cuerda pero con diferentes ángulos (45 grados, 60 grados, etc)

Presión

- Flautando

- Scratch

- Doble presión

- Sin presión

- Ruido (aleatorio)

Cambio/oscilación de altura y expresión (arco + mano izquierda)

- Vibrato sistemático

- Trino libre (trémolo)

- Glissando libre

- Scordatura libre

Posición

Articulación (pizzicato con mano derecha e izquierda)

- Staccato

- Legato

- A la guitarra

- Con yema

- Con uña

- Trémolo

- Trino

- Glissando

- M.O/Pos. Ord (Modo ordinario/ Posición ordinaria)

- S.P (Sul ponticello)

- A.S.P (Alto sul ponticello)

- S.T (Sul tasto)

- A.S.T (Alto sul tasto)

- Detrás del puente

- Detrás de la ceja

Presión

- Bártok

- Tapping

Cambio/oscilación de altura y expresión (pizzicato + mano izquierda)

- Vibrato sistemático

- Trino libre (trémolo)

- Glissando libre

- Scordatura libre 


\section{Técnicas principales (doble raíz)}

Técnicas extendidas II

fuera de las cuerdas

(Técnicas de percusión y frotación con las manos)

(T.E II)

(Principalmente usadas en los siglos xx-xxi)

\begin{tabular}{|c|c|}
\hline Articulación (golpes de arco) & $\begin{array}{l}\text { Articulación (percusión y frotación con } \\
\text { las manos y los dedos) }\end{array}$ \\
\hline - Jeté & \\
\hline - Spiccato & - Golpes simples \\
\hline - Tremolo & - Redobles \\
\hline - Arco circular & \\
\hline - Arco perpendicular & \\
\hline - Col legno & \\
\hline - Col legno battuto & \\
\hline Posición & Posición \\
\hline - En el puente & - En el puente \\
\hline (sobre el puente y los costados) & (sobre el puente y los costados) \\
\hline - En el cordal & - En el cordal \\
\hline (en la parte superior, media e inferior) & (en la parte superior, media e inferior) \\
\hline - En el puntal & - En el puntal \\
\hline (en la parte superior, media e inferior) & (en la parte superior, media e inferior) \\
\hline - En el caracol & - En el caracol \\
\hline - Sobre la ceja & - Sobre la ceja \\
\hline - En la caja de resonancia & - En la caja de resonancia \\
\hline $\begin{array}{l}\text { (sobre las partes laterales centrales } \\
\text { superiores, inferiores) }\end{array}$ & $\begin{array}{l}\text { (sobre las partes laterales centrales } \\
\text { superiores, inferiores) }\end{array}$ \\
\hline - El la tapa posterior & - El la tapa posterior \\
\hline (En la parte superior, media e inferior) & (En la parte superior, media e inferior) \\
\hline & — En el arco (sobre la madera) \\
\hline Presión & \\
\hline - Flautando & \\
\hline - Scratch & \\
\hline — Doble presión & \\
\hline — Mínima presión & \\
\hline - Ruido (sistemático) & \\
\hline
\end{tabular}




\section{Técnicas principales (doble raíz)}

Técnicas extendidas III

Relacionadas con cambio/oscilación de altura, expresión, polifonía y timbre

(T.E III)

(Principalmente usadas en los siglos XX-XXI)

\begin{tabular}{l|l} 
arco + posición de mano izquierda & $\begin{array}{l}\text { pizzicato con mano derecha + posición de } \\
\text { mano izquierda }\end{array}$ \\
- Acordes & - Acordes \\
$-\quad$ Vibrato & - Vibrato \\
$-\quad$ Trino & - Trino \\
$-\quad$ Glissando & - Glissando \\
- Armónicos naturales & - Armónicos naturales \\
- Armónicos artificiales & - Armónicos artificiales \\
- Multifónicos (de 2 a 4 sonidos) & - Multifónicos (de 2 a 3 sonidos) \\
&
\end{tabular}

Técnicas extendidas IV

Relacionadas con la posición del violonchelo

(T.E IV)

(Principalmente usadas en los siglos XX-XXI)

- Posición horizontal del violonchelo (a manera de guitarra)

- Posición vertical de pie

Tabla 1.

Principales recursos utilizados en la interpretación del violonchelo Tabla construida por el autor

Las diversas técnicas agrupadas aquí parten desde la doble raíz (arco y pizzicato), pasan por las denominadas técnicas derivadas (Td), luego por las técnicas extendidas I sobre las cuerdas (T.E I) y las técnicas extendidas II fuera de las cuerdas (T.E II). Después de clasificar dentro de las T.E II algunas técnicas de percusión y frotación que ya no están ligadas a la doble raíz, se vuelve a ella con las técnicas extendidas III (T.E III), relacionadas con oscilaciones, altura, expresión, polifonía y timbre, en las cuales la interacción simultánea de las manos derecha e izquierda es fundamental. Finalmente, esta tabla muestra algunas de las nuevas posturas físicas usadas para la interpretación del violonchelo, denominadas como técnicas extendidas IV (T.E IV).

Analizando en detalle la Tabla 1, se puede decir que algunas de estas técnicas se generan a partir de ciertas variaciones en articulación, posición, presión, expresión, altura y timbre sobre el arco y el pizzicato, además de que sus combinaciones generan características tímbricas específicas. Por ejemplo, el sonido LA - en cuerda al aireatacado con staccato, en sul ponticello y con mínima presión de arco, no tiene la misma característica sonora, si en lugar de usar mínima presión, se usa scracth. Esta última técnica generaría un alto rango de ruido que prácticamente eliminaría la audibilidad 
de la altura, mientras que el primer gesto estimularía los parciales armónicos agudos de la cuerda y la altura podría ser escuchada con más claridad. Por otro lado, cuando de las técnicas extendidas aquí indicadas se generan a partir del uso de la madera y otras partes del instrumento sin usar las técnicas fundamentales, la funcionalidad de las manos y del instrumento cambia, como cuando las manos se emplean exclusivamente para tocar el violonchelo como instrumento de percusión. Finalmente, las técnicas relacionadas con la postura física, son más bien nuevas técnicas de posición del instrumento que no solo han sido usadas con el fin de cumplir ciertas necesidades sonoras, sino también para prácticas extramusicales. Ello, no obstante, en la mayoría de los casos, requieren el uso de las técnicas primarias.

Es necesario aclarar que la Tabla 1 es el primer paso para futuras investigaciones, las cuales intentarán lograr una clasificación más profunda y detallada en cuanto a la funcionalidad de las técnicas, sus fines pedagógicos y el uso de estas como medio de exploración instrumental y creación artística.

Se podría concluir con la idea de que las técnicas extendidas en el violonchelo se desarrollaron ampliamente a partir del siglo xx, si bien fue desde los años cincuenta cuando estás técnicas empezaron a volverse más comunes dentro del repertorio para violonchelo solo, e incluso influyen a violonchelistas de géneros alternativos. Es decir, ocurre una especie de nueva estandarización técnica que no solo incluía las técnicas tradicionales usadas en el repertorio de siglos anteriores, sino que mostraba el comienzo de un desarrollo técnico alternativo, aplicado tanto en la música académica como en contextos musicales populares urbanos y underground. ${ }^{6}$ El desarrollo de las técnicas extendidas en el violonchelo generó un cambio en el universo sonoro del instrumento y en su repertorio, o sea, un sonido depurado, claro, preciso en alturas, rango dinámico y fácil de reconocer, logrado con dos técnicas fundamentales. Así, se convierte en una especie de gran paleta sonora que incluye ruido, presión, grandes oscilaciones de altura y rangos dinámicos, como algunos de sus elementos primarios. Estas nuevas características sonoras, descubiertas y potenciadas por compositores e intérpretes del siglo xx, continúan evolucionando y generando más sonoridades, estilos musicales e incluso nuevas maneras de tocar.

Muchas de las técnicas hasta aquí señaladas se han desarrollado paralelamente en otros instrumentos, no solo de cuerda frotada sino también pulsada, lo que genera un intercambio técnico que ha expandido más la funcionalidad de los medios, a la vez que ha incrementado la exigencia técnica de las piezas, al punto de llevar a los intérpretes al extremo de sus habilidades. Dos claros ejemplos de este intercambio técnico son Caja con trenzas para guitarra (2003-2004) de Julio Estrada, la cual requiere el uso de arco, y Exoskeleton (2003) for viola alla chitarra de Arthur Campella, concebida para viola, pero usándose sin arco y en posición vertical, como una guitarra.

\footnotetext{
Se fundamenta esta idea en la investigación y análisis de las piezas más importantes para violonchelo solo del siglo XX que fue publicada en una tesis doctoral en 2019. NEW TIMBRAL DIRECTIONS IN THE CONTEMPORARY CELLO REPERTOIRE: Analysis of works by Colombian composers from 2000 to 2015 , como producto en el doctorado en musicología de la Universidad de Helsinki, https://helda. helsinki.fi/handle/10138/299824?locale-attribute=en
} 
Desde la segunda mitad del siglo xx hasta el presente, el violonchelo ha pasado por varias exploraciones en su sonido y técnicas. Sin embargo, en comparación con periodos anteriores, significativamente, el timbre ha sido una de las características más exploradas. Desde las técnicas extendidas a los dispositivos electrónicos, el violonchelo ha ampliado su rango tímbrico y se ha utilizado no solo en la música clásica, sino también en casi todo tipo de música. Por otra parte, la relación simbiótica del violonchelo con las nuevas tendencias musicales ha dado lugar de alguna manera a una re-evaluación de su funcionalidad y del timbre, tanto como instrumento solista y dentro de diversos grupos. Por ejemplo, el surgimiento de la música concreta y la música electrónica alentó a compositores y ejecutantes a manipular las propiedades acústicas del instrumento, mezclándolo y procesándolo con dispositivos electrónicos. El indeterminismo y el experimentalismo condujeron a su vez a la exploración de parámetros cuya relación con el azar amplió la paleta sonora instrumental. El uso del violonchelo, no solo como instrumento musical, sino como generador de sonidos, puede atribuirse a la influencia de las artes visuales y el ruido. La música experimental improvisada generó innovaciones radicales del timbre en el medio, ya que los compositores y ejecutantes trabajaron principalmente con procedimientos experimentales aplicados a técnicas extendidas, dispositivos electrónicos, ruido y objetos sonoros, entre otros. A diferencia de los compositores e intérpretes experimentales, los violonchelistas de jazz, rock y pop no parecen centrarse en de-construir el sonido del instrumento hasta el punto de hacerlo irreconocible; por el contrario, han buscado un punto de convergencia entre el sonido tradicional y el procesado del instrumento. El conjunto de observaciones anteriores permite afirmar que el violonchelo ha adquirido una nueva identidad, misma que continúa desarrollándose y mutando constantemente. Incluso, la construcción del violonchelo contemporáneo ha adquirido nuevas formas, materiales y funciones, lo cual sigue contribuyendo al desarrollo de nuevas tendencias musicales y de los nuevos intérpretes. 


\section{Referencias}

\subsection{Bibliografía}

Castrillón Arcila, S. A. (2016). Understanding Performance with Electronics and Beyond: Historical Overview, Significant pieces and the Influence of Visual and Performing Arts in the Repertoire for Cello with Electronics and Mixed Media. Ideas Sónicas, 9(7).

Castrillón Arcila, S. A. (2019). New Timbral Directions In The Contemporary Cello Repertoire: Analysis of works by Colombian composers from 2000 to 2015. Editorial Unigrafia, Facultad de Artes, Universidad de Helsinki.

Homuth, D. (1994). Cello Music since 1960: A Biography of Solo Cello, Chamber and Orchestral works for the Solo Cellist. Berkeley: Fallen Leaf Press.

Markevitch, D. (1989). The Solo cello: A Biography of the Unaccompanied Violonchelo Literature. Berkeley: Fallen Leaf Press.

Stowell, R. (2000). "Solo Cello Repertoire". En R. Stowell (Ed.), The Cambridge companion to the Cello (pp. 137-145). Cambridge: Cambridge University Press.

Uitti, F. M. (2000). “The Frontiers of Technique”. En R. Stowell (Ed.), The Cambridge companion to the Cello (pp. 211-223). Cambridge: Cambridge University Press.

\subsection{Fuentes primarias}

Cassadó, G. (1926). Suite per violonchelo. London: Universal Edition.

Castrillón Arcila, S. A. (2014). Yo soy la selva. Obra Inédita.

Dallapiccola, L. (1947). Ciaccona, Intermezzo e Adagio per violonchelo solo. Wien: Universal Edition.

Kodály, Z. (1921). Sonata para violonchelo solo. London: Universal Edition.

Lachenmann, H. (XXX). Pression for one cellist. Leipzig: Breitkopf \& Hartel.

Saaraiho, K. (1989). Petals. Helsinki: Edition Wilhem Hansen.

Scelsi, G. (1960). Trilogy. Roma: Edizioni dell’autore.

Xenakis, I. (1977). Kottos. Paris: Editions Salabert.

Zimmermann, B. A. (1961). Sonate für cello solo. München: Edition Modern. 